\title{
Micro-macro acoustic modeling of heterogeneous foams with nucleation perturbation
}

\author{
Cong Truc NGUYEN ${ }^{\mathrm{a}, \mathrm{b}}$, Johann GUILLEMINOT ${ }^{\mathrm{c}}$, Fabrice DETREZ ${ }^{\mathrm{a}}$, Vincent LANGLOIS ${ }^{\mathrm{d}}$, Michel BORNERT ${ }^{\mathrm{e}}$, Arnaud DUVAL $^{\mathrm{b}}$ and Camille \\ PERROT $^{\mathrm{a}, *}$
}

\author{
${ }^{a}$ MSME, Univ Gustave Eiffel, CNRS UMR 8208, Univ Paris Est Creteil, F-77454 Marne-la-Vallée, France \\ ${ }^{b}$ Trèves products, services and innovation, 2-4 rue Emile Arquès, CS 70017, 51686 Reims Cedex 2, France \\ ${ }^{c}$ Department of Civil and Environmental Engineering, Pratt School of Engineering, Duke University, P.O. Box 90287, 121 Hudson Hall, Durham, North Carolina \\ 27708-0287, USA \\ ${ }^{d}$ Navier, Univ Gustave Eiffel, ENPC, CNRS, IFSTTAR, F-77447 Marne-la-Vallée, France \\ e Navier, Univ Gustave Eiffel, ENPC, CNRS, F-77447 Marne-la-Vallée, France \\ * The author to whom any correspondence should be addressed. E-mail: camille.perrot@univ-eiffel.fr (C. Perrot).
}

\begin{abstract}
The properties of a polyurethane foam are greatly influenced by the addition of graphite particles during the manufacturing process, initially used as a fire retardant. These thin solid particles perturbate the nucleation process by generating bubbles in their immediate vicinity. A large body of work has focused on foams that are reasonably homogeneous. In this work, we propose a modeling approach for inhomogeneous foams that includes membrane effects and allows pore size distributions to be accounted for. The cellular structure of the foam is obtained through a random Laguerre tessellation optimized from experimental properties. The structure of real foam samples is analyzed using X-ray computed tomography and scanning electron microscopy, followed by image processing, to create three-dimensional, digital models of the samples. The corresponding effective material parameters, including the permeability, the tortuosity and the viscous characteristic length, are subsequently computed by applying a numerical homogenization approach. All the numerical data are presented, discussed and gauged against experimental results.
\end{abstract}

\section{Introduction}

Polyurethane (PU) foams generally present a disordered structure with a random distribution of pore size and are utilized in many applications where acoustical properties are required. The study of its acoustical properties has been carried out by several works, in which the link between acoustical properties and microstructural parameters have been predicted by different geometry models $[20,2,19]$. It has been shown that the presence of membranes is a key parameter to control acoustic absorption capacity of foams; and that both i) the proportion of closed membranes and ii) the closure ratio of open membranes must be taken into account in the model $[2,20]$. However, the previous works have been carried out with the constant pore size using essentially combinations and appropriate averaging approaches based on idealized periodic unit cells (PUCs). Therefore, the major objective

${ }^{1}$ https://www.mecanum.com/porosimetre-densimetre?lang=en of this paper is to study the permeability and acoustic properties of anisotropic and/or locally inhomogeneous foams.

In this study, we focus on the influence of pore size distribution and membrane effect on the acoustic properties of PU foams. The pore size distribution and the pore anisotropy are characterized from X-Ray micro-tomography imaging. The membranes content is evaluated by the fraction of closed membranes and the aperture ratio of open membranes from scanning electron microscope images. A threedimensional (3D) structural model based on a polyhedral description of the foam is achieved by determining appropriate attributes of the seeds of Laguerre tessellations from experimental properties. The viscous and thermal permeabilities, tortuosity, viscous and thermal characteristic lengths computed along the $x, y$, and $z$ axis perpendicular to the cubic unit cell boundaries in which the polyhedra are generated. This provides the transport properties for the calculation of the sound absorption coefficient of the PU foam samples derived using the semiphenomenological JCAL model [3,5]. Finally, the computed viscous permeability and sound absorption coefficients are compared with three-microphone impedance tube acoustical measurements.

\section{Characterization of the foam samples}

\section{Porosity and permeability}

The open porosity $\phi$ of a foam is defined as the ratio of the interconnected pore volume to the total bulk volume of the foam domain. It is an important geometrical parameter, which relates the properties of the air saturating in the interconnected pores to the effective properties of the foam [1]. In this work, the open porosity was measured by the pressure/mass method, which is based on the ideal gas law, developed by L. Beranek and further elaborated by Y. Salissou and R. Panneton [16]. The measurement was carried out at CERA Centre d'Etudes et de Recherche Automobile (Reims) with the system of porosity and density meter Phi-X. ${ }^{1}$

The permeability $k_{0}\left(\mathrm{~m}^{2}\right)$ of the foam sample is determined by the measurement of the airflow resistivity $\sigma\left(\mathrm{Ns} / \mathrm{m}^{4}\right)$ through the relation $\sigma=\eta / k_{0}$ where $\eta$ is the viscosity of the air saturating in the

Page 1 of 6

$12 / 11 / 2020$ 
interconnected pores. The resistivity is measured by a measurement of the pressure drop depending on volumetric airflow rate as described in DIN EN 29053 (ISO 9053) with an airflow resistivity meter AFD 300 - AcoustiFlow ${ }^{2}$ at MSME - Multi Scale Modeling and Simulation laboratory (Marne-la-Vallée).

The polyurethane foam investigated in this study exhibits a high open porosity value, $\phi=0.986 \pm 0.001$; and low permeability value, $k_{0}=$ $(1.91 \pm 0.21) \times 10^{-10}\left(\mathrm{~m}^{2}\right)$. These somehow contradictory values suggest that the membrane and/or pore size distribution effects are strong.

\section{Pore size distribution}

The distributions of pore size of the foam samples are obtained from $\mathrm{X}$-Ray computed micro-tomography (X-ray $\mu \mathrm{CT}$ ), a non-destructive method enabling to observe the foam cross-sectional cuts in the micrometer range. Due to the poor X-ray mass attenuation coefficients of the PU foam under study, the thin membranes essentially disappear in the corresponding $\mu \mathrm{CT}$ images, the pores are usually connected through the fluid phase. For further morphological analysis, the pores have to be separated as individual ones. Following Nistor et al., a proper morphological characterization of the foam is performed from the 3D porous structure of the PU foam reconstructed using tomography cross-section images acquired at the F2M microtomography platform available at Navier laboratory [10]. Four main steps are required (Fig. 1):

1. First, the foam struts must be obtained through binarization and noise filtering of the obtained images.

2. In the second step; a distance transform map of individual pores is computed together with an inversion of the images from the first step.

3. Then, a watershed transform allows separating one pore from another.

4. Finally, the foam surroundings are deleted.

When the procedure described above is performed as illustrated in Fig. 1 , the distribution of the pore size can be estimated from the $3 \mathrm{D}$ reconstructed foam. In the pore size distribution, the pore size is represented by a so-called equivalent diameter $d$, which represents the diameter of a sphere with a volume equivalent to the volume of the corresponding pore. From now on, and unless otherwise stated, the normalized pore size will be referred to as $d /\langle d\rangle$, where $\langle d\rangle$ is the average diameter over all pores. Also, worth to be mentioned is that the performed procedure also provides with the relative position of each pore, together with the corresponding neighboring pores.

The sphericity index $s$, which can be defined as the ratio of the surface area of the sphere of equivalent volume over the surface area $S$ of the corresponding pore, is another interesting shape parameter. The sphericity index takes a maximal value of one for a spherical pore and it will take small values if the pore shape differs significantly from the shape of a sphere. This sphericity index is defined by,

2

https://www.akustikforschung.de/en/produkte/messgerate/stromungs widerstandsmessgerat-aed-300-acoustiflow/

$$
s=\left(36 \pi \frac{V^{2}}{S^{3}}\right)^{1 / 3}
$$

and is applicable to represent the anisotropic character of the pores.

All image processing and analysis steps were carried out by using the open-source FIJI software with the plugins MorphoLibJ $[7,18]$.
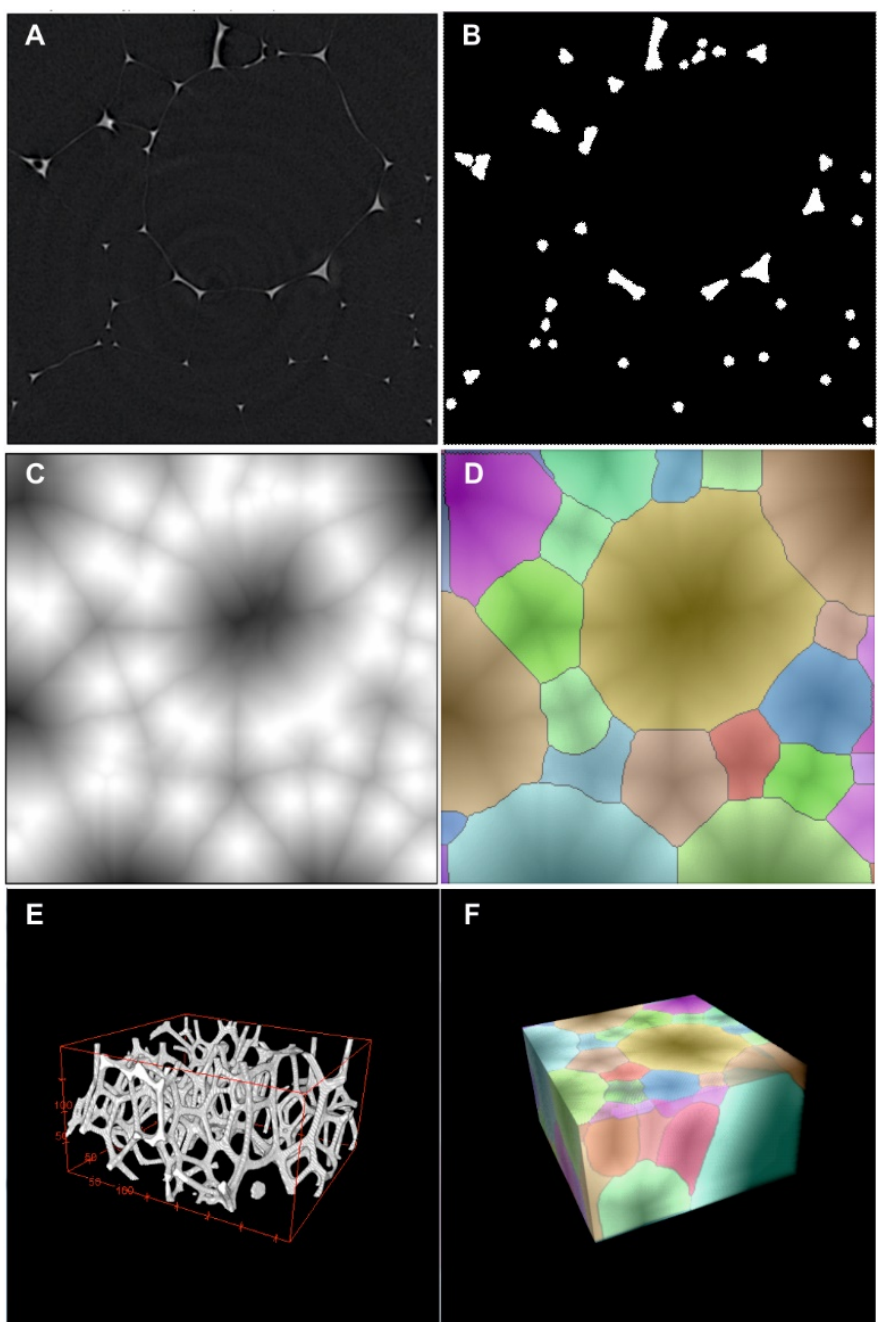

$\mathbf{F}$

Figure 1. Detection and pore size distribution from X-ray $\mu \mathrm{CT}$ and image analysis. A) Original image of micro-tomography. B) Binarized image generating the struts system of foam. C) Image obtained after distance transform and inversion. D) Detected pores after segmentation by watershed transform. E \& F) 3D visualization of struts system and the corresponding detected pores.

From the procedure described above, a parallelepipedic field of view of $6 \times 6 \times 6 \mathrm{~mm}^{3}$ containing 2131 pores was analyzed with a voxel size corresponding to $6 \mu \mathrm{m}$. The distribution of pore size together with the complement of the sphericity index, $1-s$, are plotted in Fig. 4. 
Furthermore, the porosity is also estimated, by considering that the solid part of the foams is mainly concentrated in the struts system, through the binarized image (see Fig. 1B \& E). The proportion of solid part is equal to $1.37 \%$. Accordingly, the porosity is equal to $98.63 \%$, a value in full agreement with the data independently obtained from the pressure/mass measuring method. We note that the small difference is in agreement with the fact that the membranes are not appearing in the $3 \mathrm{D}$ reconstructed foam.

\section{Membrane characteristics}

The characterization of membranes is performed by analyzing the surfaces of the foam sample through scanning electron microscope (SEM) images. A polygon is superimposed over each individual window. For each opened membrane, an ellipse is used to characterize the area of the corresponding aperture size (see Fig. 2). Then, the area of the superimposed polygons and of ellipses are determined. The proportion of closed and open membranes, respectively denoted by $x_{c}$ and $x_{o}$, are also reported. The aperture ratio of open membranes is further estimated as $t_{o}=\sqrt{A_{e l} / A_{p l}}$, where $A_{p l}$ is the area of the polygon corresponding to a window for which the membrane is open and $A_{e l}$ is the area of the ellipses associated with the given aperture. Then the average of aperture ratio $\left\langle t_{o}\right\rangle$ is deduced.

For the PU foam under study, several tens SEM images of both the top and bottom sample surfaces analyzed, a mean value for the proportion of closed membranes $\left\langle x_{c}\right\rangle=0.76$ is observed, and the average of aperture ratio for the open membranes $\left\langle t_{o}\right\rangle=0.17$ is obtained.

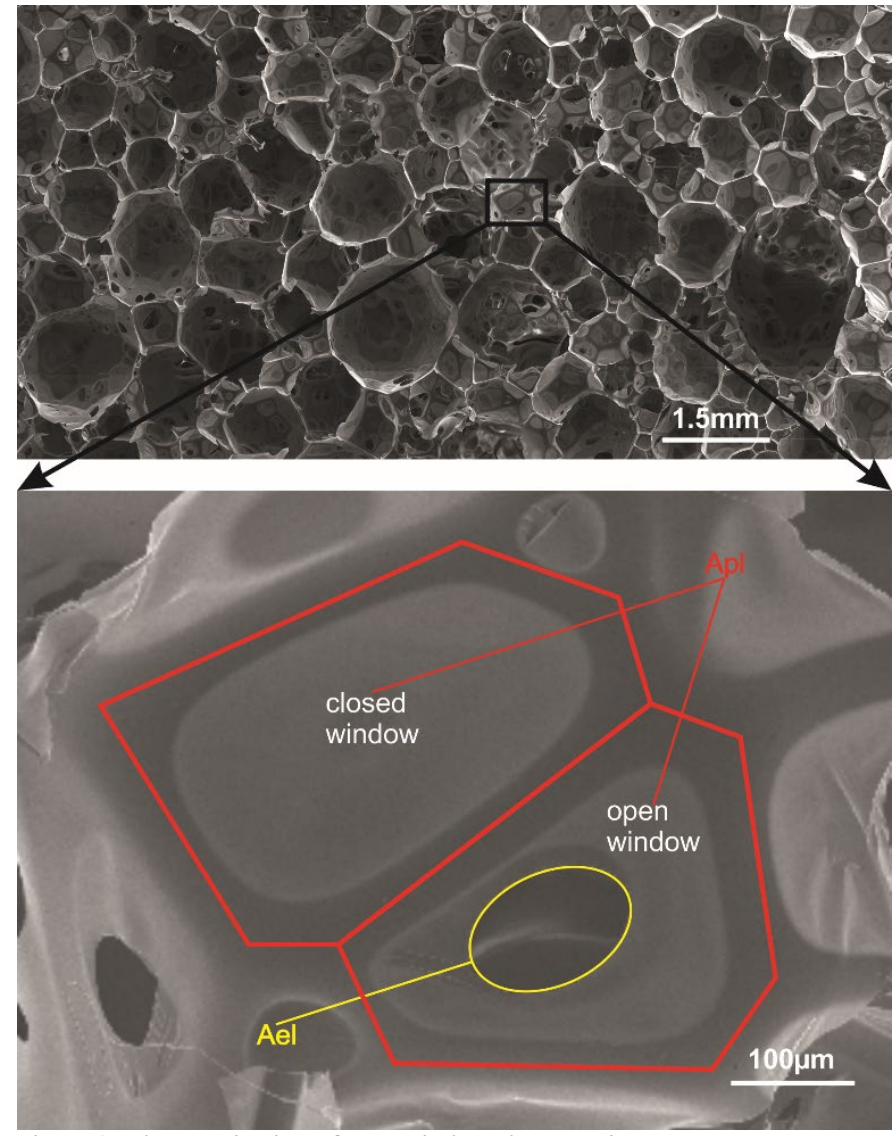

Figure 2. Characterization of pore windows by SEM image.
The thickness of the membrane is also estimated through SEM images. Its average value is in the range $1-2 \mu \mathrm{m}$, in agreement with the membrane thickness of polyurethane foams found in other works [2, $20]$. One can note that the membrane thickness is very thin when compared to the average pore size $\langle d\rangle=360 \mu \mathrm{m}$. This property will be used later on to justify that the thickness of the walls separating cells can be ignored in order to reduce the size of the finite element model.

\section{Acoustic measurements}

A three-microphone impedance tube (length, $1 \mathrm{~m}$; diameter, $40 \mathrm{~mm}$ ) was used to characterize the acoustic properties of the PU foam under study. Based on this experimental setup, (i) the sound absorption coefficient at normal incidence $\left(S A C_{N I}\right)$ is directly measured, and (ii) supplementary frequency-dependent response functions of the porous material modeled as an equivalent fluid are determined from the pressure transfer functions between the microphones [ (Salissou et Panneton 2010)], i.e., equivalent density $\tilde{\rho}_{e q}$ and equivalent bulk modulus $\widetilde{K}_{e q}$.

\section{Numerical simulations}

\section{Generation of the foam structures}

Random Laguerre tessellation are widely used to simulate the pore structures of polydisperse foams $[15,8,21]$. In these works, the Laguerre tessellations were essentially based on a random dense packing of hard spheres, which have a distribution of sphere sizes coinciding with the pore size distributions of foam samples. In this case, large differences between the spherical and pore centroids can be obtained and therefore, the quantitative reproduction of the pore size distribution found in experimental data is rather poor. Recently, an improved algorithm of Laguerre tessellations has been developed [14]. The idea is to consider the desired (experimental) pore properties as an input of the problem, from which the attributes of the seeds are determined by updating tessellations between iterations from a generic algorithm (until an optimal configuration is found). It is also recommended to replace small edges and faces by single vertices, a correction method called regularization such that the tessellations can further be meshed with good finite element conditioning. Tiny facets are neglected when $\lambda<\epsilon\langle\lambda\rangle$, where $\lambda$ is the facet area, $\langle\lambda\rangle$ is the average area and $\epsilon$ is the threshold that restricts the facets surface area. Typically, $0.01 \leq \epsilon \leq 0.10$ [4]. In this work, $\epsilon=0.05$ corresponds to the facets surface area threshold used for regularization.

A structure of 242 pores is generated to mimic the pore size distribution of the PU foam under study. It is assumed that the pore size follows a lognormal probability law, with mean $0.36 \mathrm{~mm}$ and standard deviation $0.29 \mathrm{~mm}$. The sphericity is also assumed to follow a lognormal distribution with a $1-s$ lognormal distribution of average 0.23 and standard deviation 0.068 . The average proportion of open membranes $\left\langle x_{o}\right\rangle$ and the average of aperture ratio for the open membranes $\left\langle t_{o}\right\rangle$ are taken into account in the model as follows, see Fig. 3: (i) the open membranes are randomly selected among all the membranes of the model, (ii) among the membranes randomly selected to be opened, the membranes are locally opened by a circle with an aperture ratio equal to the average of aperture ratio for the open membranes. 

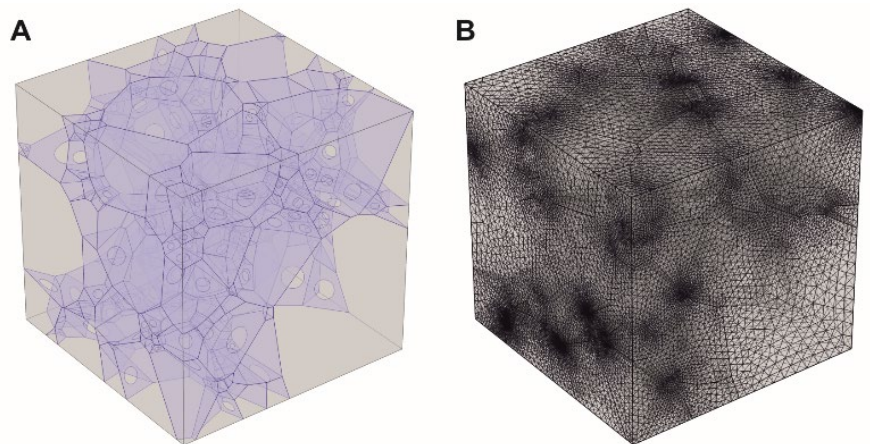

Figure 3. Generated geometry (A) and corresponding mesh with 7360100 tetrahedral elements (B).

\section{Calculation of acoustical properties}

The acoustical properties of the equivalent fluid characterizing viscothermal dissipation mechanisms within the porous structure supposed to be motionless can be determined from the calculation of two complex response functions, the equivalent density $\tilde{\rho}_{e q}$ characterizing the viscous-inertial effects and equivalent bulk modulus $\widetilde{K}_{e q}$ associated with the thermal exchanges. Then, the equivalent wave number $\tilde{k}_{e q}$ and equivalent characteristic impedance $\tilde{Z}_{e q}$ directly follows from analytical calculations.

For rigidly backed porous material of thickness $L_{S}$, the sound absorption coefficient $S A C_{N I}$ at normal incidence is given by:

$$
S A C_{N I}=1-\left|\frac{Z_{s}-\rho_{0} c_{0}}{Z_{s}+\rho_{0} c_{0}}\right|^{2}, \quad \tilde{Z}_{s}=-i \tilde{Z}_{e q} / \phi \cot \left(\tilde{k}_{e q} L_{s}\right)
$$

where $\rho_{0}$ is the density of air at rest, $c_{0}$ is the sound speed in air and $\tilde{Z}_{s}$ is the normal incidence surface impedance.

Based on the JCAL semi-phenomenological model, all of the appropriate response functions can be derived from the basis of transport parameters. The set of six transport parameters used $\left(\phi, \Lambda^{\prime}, k_{0}, \Lambda, \alpha_{\infty}, k_{0}^{\prime}\right)$ is computed by the numerical homogenization approach. More precisely, the porosity $\phi$ and the thermal characteristic length $\Lambda^{\prime}$ are obtained from geometry, and other transport parameters are computed numerically from appropriate field averaging from three boundary value problems. The static viscous permeability $k_{0}$ is derived from the Stoke equations; the tortuosity $\alpha_{\infty}$ and the viscous characteristic length $\Lambda$ are computed from the potential flow equations; Laplace equation of thermal diffusion enables the computation of the static thermal permeability $k_{0}^{\prime}$. The details on this numerical homogenization approach were described in various works [13]. These boundary value problems were solved by the finite element method using the commercial software COMSOL Multiphysics. The calculation is performed on three different configurations and along the $x, y$, and $z$ axis perpendicular to the cubic unit cell boundaries in which the pores are generated.

\section{Results and discussion}

\section{Distributions of pore size and of pore sphericity}

Figure 4 shows the comparison between the experimental microstructure and the simulated microstructure in terms of both the distributions of pore size and spheriticity $s$ (plotted as $1-s$ ) together with the corresponding lognormal fits.
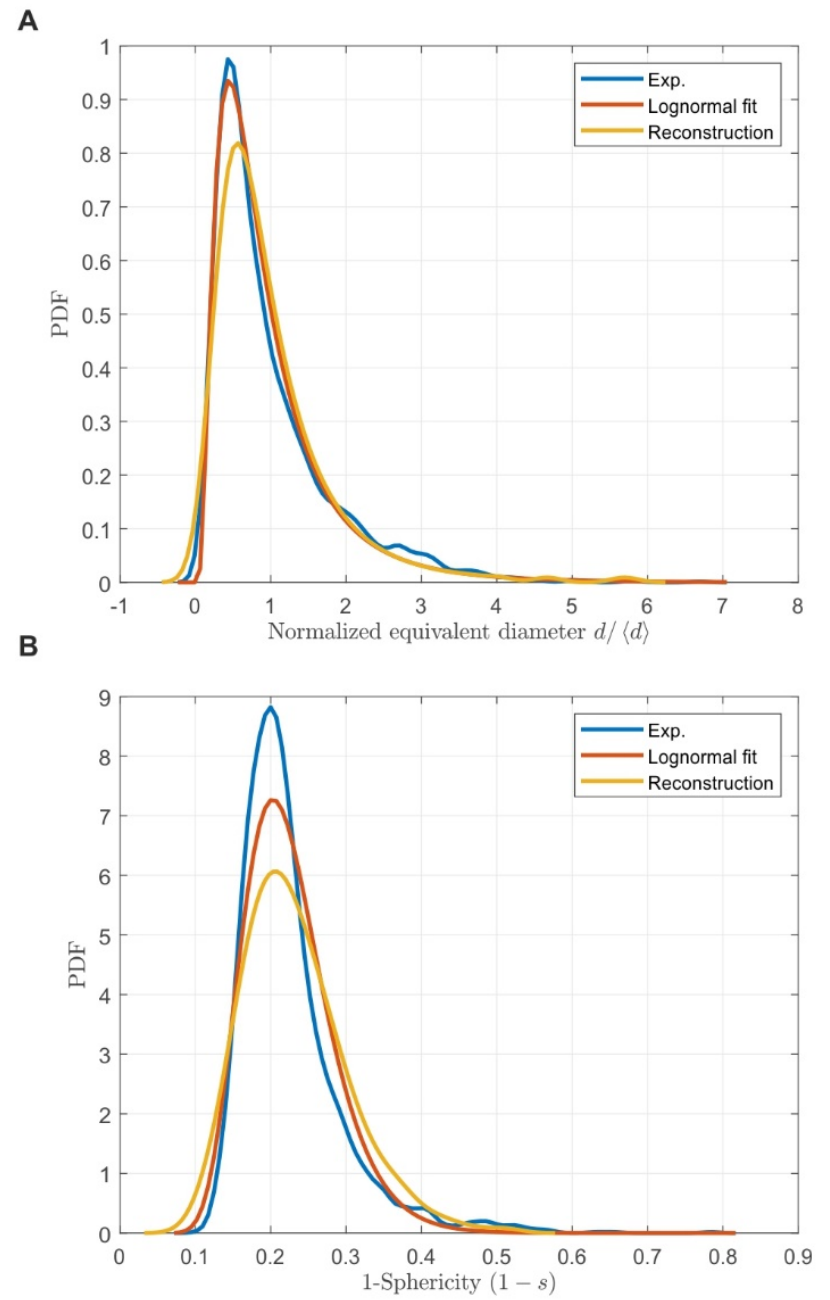

Figure 4. Foam morphology comparison between experimental measure, fitted lognormal law and reconstruction for (A) pore size distribution and (B) 1sphericity.

The high value of the variation coefficient for the pore size $c=\sigma_{d} /\langle d\rangle$ $=0.79$, with $\sigma_{d}$ denoting the standard deviation of the pore size $d$, denotes a wide distribution of pore sizes. The lognormal fit of the pore size distribution is clearly in agreement with the experimental microstructure, in agreement with previously reported studies of pore sizes in cellular materials [15]. Similarly, a lognormal fit is employed to extract the statistical characteristics of the sphericity of the pores. Considering the relatively low number of pores used to simulate the experimental microstructure together with a wide distributions of pore size and sphericity, the cell diameter and sphericity distributions adequately match the prescribed distributions (Fig. 4).

Moreover, the average number of neighboring pores for the simulated microstructure, 9.6, is also close to the value obtained for the experimental microstructure, 9.1. It is worth to be mentioned that this 
value is exceptionally low, when compared to typical monodisperse foams for which the typical values lies in the range of $12-14[8,21$, 9]. This exceptional feature of the PU foam under study is to be related primarily to the wide distribution of pore sizes: local inhomogeneity of the pore sizes is obtained as a result of the particular foaming process (small pores have fewer neighbors than larger ones). This tendency is increased by the statistical anisotropy of the pores.

\section{Acoustical properties}

The main objective of this section is to produce a comparison between the viscous permeability and the sound absorption coefficients determined from experimental measurements and multi-scale numerical simulations. Note that the remaining transport parameters can be estimated experimentally using an inverse characterization method proposed by Panneton and Olny [12,11], from the threemicrophone tube measurements. In context of this work, due to time limitations, we the focus is on the viscous permeability. The computed values of the transport parameters are listed in Table I. It should be mentioned that the porosity used for the calculations of the equivalent fluid properties is the one given by the experimental value. The solution fields corresponding to the simulated microstructure are shown in Fig. 5, together with a comparison of the sound absorption coefficient at normal incidence for the experimental and simulated microstructures. A strong anisotropy of the transport properties is induced by the pore size and shape inhomogeneity. In average, for the three-different simulated microstructures, the computed viscous permeability $1.60 \pm 1.38 \times 10^{-10} \mathrm{~m}^{2}$ is in agreement with measured value $1.91 \pm 0.21 \times 10^{-10} \mathrm{~m}^{2}$. Once again, the variability of the simulated permeability is due to the relatively low number of simulated pores. Figure 5D show that the computed sound absorption coefficient is in line with measurements, without any adjusted parameter. We note that the presence of membranes reduces the foam sample permeability and favors a strong coupling between the fluid and the solid phase of the foam, which also explains the observed variability in the sound absorption measurements. Also of great interest are the high values of tortuosity, simulated for the first time in agreement with preliminary experimental observations for such kind of foams.

Table 1. Computed transport parameters. $\left(k_{0}\left[\times 10^{-10} \mathrm{~m}^{2}\right], k_{0}^{\prime}\left[\times 10^{-8} \mathrm{~m}^{2}\right]\right.$, $\left.\Lambda, \Lambda^{\prime}[\mu m]\right)$

\begin{tabular}{|c|c|c|c|c|c|c|c|c|c|c|}
\hline & \multicolumn{3}{|c|}{ Config. 1 } & \multicolumn{3}{c|}{ Config. 2 } & \multicolumn{3}{c|}{ Config. 3 } & \multirow{2}{*}{ Ave. } \\
\hline & $x$ & $y$ & $z$ & $x$ & $y$ & $z$ & $x$ & $y$ & $z$ & \\
\hline$\phi$ & \multicolumn{8}{|c|}{$\mathbf{0 . 9 8 6}$} \\
\hline$\Lambda^{\prime}$ & \multicolumn{3}{|c|}{662.7} & \multicolumn{3}{c|}{661.0} & \multicolumn{3}{c|}{663.7} & $\mathbf{6 6 2 . 5}$ \\
\hline$k_{0}$ & 0.39 & 0.68 & 4.25 & 1.00 & 3.07 & 0.56 & 0.40 & 2.53 & 1.50 & $\mathbf{1 . 6 0}$ \\
\hline$\alpha_{\infty}$ & 5.68 & 5.90 & 2.44 & 4.43 & 3.06 & 5.04 & 4.33 & 2.96 & 3.05 & $\mathbf{4 . 1 0}$ \\
\hline$\Lambda$ & 138.9 & 186.1 & 258.3 & 175.9 & 101.8 & 144.2 & 123.4 & 130.5 & 174.2 & $\mathbf{1 5 9 . 3}$ \\
\hline$k^{\prime}{ }_{0}$ & \multicolumn{3}{|c|}{6.51} & & \multicolumn{7}{c|}{6.52} & \multicolumn{5}{c|}{6.56} & $\mathbf{6 . 5 3}$ \\
\hline
\end{tabular}
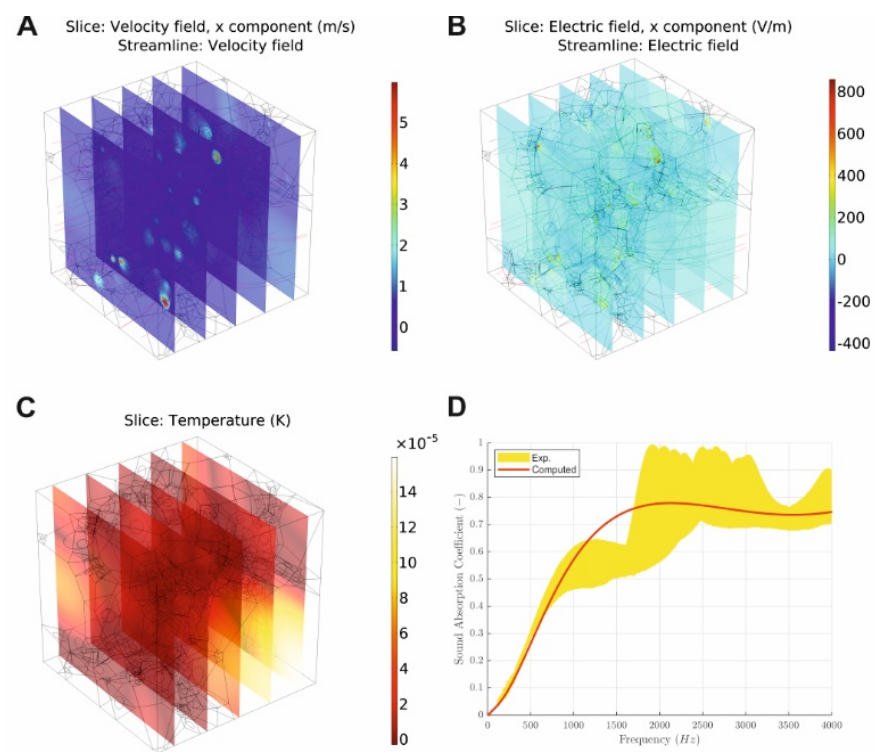

Figure 5. Solution field: (A) Stoke flow. (B) Potential flow. (C) Thermal field. (D) Sound absorption coefficient $\left(S A C_{N I}\right)$. Sample thickness: $21 \mathrm{~mm}$.

\section{Conclusions}

A three-dimensional model of heterogeneous foams including a wide distribution of pore size and sphericity was obtained to predict the acoustical properties of a heterogeneous polyurethane foam with highporosity manufactured following a nucleation perturbation technique. The heterogeneity and anisotropy of foam sample were taken into account by means of the distributions of pore size and pore sphericity used as input parameters. These experimental distributions obtained from the X-Ray micro-tomography images were fitted by two lognormal laws. The 3D geometry model uses an advanced Laguerre tessellation algorithm in which the membrane thickness was neglected and the facets smaller than a given critical threshold removed to increase the quality of the mesh subsequently used in the finite element method. The proportion of closed membranes and the aperture ratio of open membranes were measured through SEM images and included in the model. The determination of the transport parameters of the modeled microstructures followed from numerical homogenization, from which the sound absorption coefficient at normal incidence was derived. Despite the variability of the computed permeability of the simulated microstructures, the average value of the computed permeability was found to agree with the experimental one, which supports the validation of the proposed model. Additional work is underway for a better validation of the heterogeneous foam model. It includes a detailed examination of the experimental values of the transport parameters, modelling microstructures with a larger number of pores, simulating a large number of heterogeneous configurations.

\section{References}

1. Biot, Maurice A. 1956. "Theory of propagation of elastic waves in a fluid-saturated porous solid. II. Higher frequency range." The Journal of the acoustical Society of america (ASA) 28: 179191. doi:10.1121/1.1908241.

2. Gao, K., J. A. W. Dommelen, and M. G. D. Geers. 2016.

"Microstructure characterization and homogenization of acoustic polyurethane foams: Measurements and simulations." International Journal of Solids and Structures (Elsevier) 100: 536-546. doi:10.1016/j.ijsolstr.2016.09.024.

3. Johnson, David Linton, Joel Koplik, and Roger Dashen. 1987. "Theory of dynamic permeability and tortuosity in fluid- 
saturated porous media." Journal of fluid mechanics (Cambridge University Press) 176: 379-402.

doi:10.1017/s0022112087000727.

4. Kraynik, Andrew M., Douglas A. Reinelt, and Frank Swol. 2003. "Structure of random monodisperse foam." Physical Review E (APS) 67: 031403. doi:10.1103/physreve.67.031403.

5. Lafarge, Denis, Pavel Lemarinier, Jean F. Allard, and Viggo Tarnow. 1997. "Dynamic compressibility of air in porous structures at audible frequencies." The Journal of the Acoustical Society of America (ASA) 102: 1995-2006. doi:10.1121/1.419690.

6. Langlois, V., V. H. Trinh, C. Lusso, C. Perrot, X. Chateau, Y. Khidas, and O. Pitois. 2018. "Permeability of solid foam: Effect of pore connections." Phys. Rev. E (American Physical Society) 97 (5): 053111. doi:10.1103/PhysRevE.97.053111.

7. Legland, David, Ignacio Arganda-Carreras, and Philippe Andrey. 2016. "MorphoLibJ: integrated library and plugins for mathematical morphology with ImageJ." Bioinformatics 32: 3532-3534. doi:10.1093/bioinformatics/btw413.

8. Liebscher, A., C. Proppe, C. Redenbach, and D. Schwarzer. 2013. "Stochastic Multiscale Modeling of Metal Foams." Procedia IUTAM 87-96. doi:10.1016/j.piutam.2013.01.010.

9. Montminy, Matthew D., Allen R. Tannenbaum, and Christopher W. Macosko. 2004. "The 3D structure of real polymer foams." Journal of Colloid and Interface Science 280: 202-211. doi:10.1016/j.jcis.2004.07.032.

10. Nistor, Andra, Miloš Toulec, Alexandr Zubov, and Juraj Kosek. 2016. "Tomographic reconstruction and morphological analysis of rigid polyurethane foams." Macromolecular Symposia. 87-95.

11. Olny, Xavier, and Raymond Panneton. 2008. "Acoustical determination of the parameters governing thermal dissipation in porous media." The Journal of the Acoustical Society of America (ASA) 123: 814-824. doi:10.1121/1.2828066.

12. Panneton, Raymond, and Xavier Olny. 2006. "Acoustical determination of the parameters governing viscous dissipation in porous media." The Journal of the Acoustical Society of America (ASA) 119: 2027-2040. doi:10.1121/1.2169923.

13. Perrot, Camille, Fabien Chevillotte, Minh Tan Hoang, Guy Bonnet, François-Xavier Bécot, Laurent Gautron, and Arnaud Duval. 2012. "Microstructure, transport, and acoustic properties of open-cell foam samples: Experiments and three-dimensional numerical simulations." Journal of Applied Physics (AIP) 111: 014911. doi:10.1063/1.3673523.

14. Quey, Romain, and Loïc Renversade. 2017. "Optimal polyhedral description of 3D polycrystals: method and application to statistical and synchrotron X-ray diffraction data."

15. Redenbach, Claudia. 2009. "Microstructure models for cellular materials." Computational Materials Science (Elsevier) 44: 1397-1407. doi:10.1016/j.commatsci.2008.09.018.

16. Salissou, Y., and R. Panneton. 2007. "Pressure/mass method to measure open porosity of porous solids." Journal of applied physics (AIP) 101: 124913. doi:10.1063/1.2749486.

17. Salissou, Yacoubou, and Raymond Panneton. 2010. "Wideband characterization of the complex wave number and characteristic impedance of sound absorbers." The Journal of the Acoustical Society of America (ASA) 128: 2868-2876.

18. Schindelin, Johannes, Ignacio Arganda-Carreras, Erwin Frise, Verena Kaynig, Mark Longair, Tobias Pietzsch, Stephan Preibisch, et al. 2012. "Fiji: an open-source platform for biological-image analysis." Nature methods (Nature Publishing Group) 9: 676.

19. Tan Hoang, Minh, and Camille Perrot. 2013. "Identifying local characteristic lengths governing sound wave properties in solid foams." Journal of Applied Physics (AIP) 113: 084905. doi:10.1063/1.4793492.

20. Trinh, Van Hai, Vincent Langlois, Johann Guilleminot, Camille Perrot, Yacine Khidas, and Olivier Pitois. 2019. "Tuning membrane content of sound absorbing cellular foams: Fabrication, experimental evidence and multiscale numerical simulations." Materials \& Design (Elsevier) 162: 345-361. doi:10.1016/j.matdes.2018.11.023.

21. Vecchio, Irene, Claudia Redenbach, Katja Schladitz, and Andrew M. Kraynik. 2016. "Improved models of solid foams based on soap froth." Computational Materials Science (Elsevier) 120: 60-69. doi:10.1016/j.commatsci.2016.03.029.

\section{Acknowledgments}

This work was part of a project supported by ANRT and Trèves Group's Automotive Investigation \& Research Center under convention CIFRE No. 2017/1908. The F2M is also acknowledged. 